\title{
Metabolic consequences of a $50 \%$ partial pancreatectomy in humans
}

\author{
B. A. Menge $\cdot$ H. Schrader • T. G. K. Breuer • \\ Y. Dabrowski • W. Uhl • W. E. Schmidt • J. J. Meier
}

Received: 6 September 2008 / Accepted: 5 October 2008 / Published online: 27 November 2008

(C) Springer-Verlag 2008

\begin{abstract}
Aims/hypothesis Partial pancreatectomy is frequently performed in patients with pancreatic tumours or chronic pancreatitis, but little is known about the metabolic impact of this intervention. We examined the effects of approximately $50 \%$ partial pancreatectomy on glucose homeostasis and insulin secretion.

Methods Fourteen patients with chronic pancreatitis, ten patients with pancreatic carcinoma and 13 patients with benign pancreatic tumours or extra-pancreatic masses (control group) underwent $240 \mathrm{~min}$ oral glucose tolerance tests before and after pancreatic tail-resection $(n=12)$, duodenopancreatectomy $(n=19)$ or duodenum-preserving pancreatic-head resection $(n=6)$.

Results Partial pancreatectomy led to a reduction in postchallenge insulin excursions by $49 \%$ in chronic pancreatitis patients, $52 \%$ in carcinoma patients and $55 \%$ in controls $(p<0.05)$. Nevertheless, post-challenge glucose concentrations were transiently ameliorated after surgery $(p<0.001)$. In the control participants, pancreatic-head resection caused a transient reduction of post-challenge glycaemia, whereas pancreatic-tail resection increased both fasting and post-
\end{abstract}

B. A. Menge and H. Schrader contributed equally to this study

B. A. Menge • H. Schrader - T. G. K. Breuer ' Y. Dabrowski •

W. E. Schmidt • J. J. Meier $(\triangle)$

Department of Medicine I, St Josef-Hospital,

Ruhr-University Bochum,

Gudrunstr. 56,

44791 Bochum, Germany

e-mail: juris.meier@rub.de

W. Uhl

Department of Surgery, St Josef-Hospital,

Ruhr-University Bochum,

Bochum, Germany challenge glycaemia $(p<0.05)$. Insulin sensitivity was highest in chronic pancreatitis patients before surgery $(p<0.01)$, but remained unchanged by the partial pancreatectomy. High pre-operative body weight and elevated fasting glucose levels were associated with poor glycaemic control after surgery. Conclusions/interpretation Insulin secretion is diminished after pancreatic-head and -tail resection, but post-challenge glucose concentrations can be ameliorated after pancreatichead resection. These data highlight the unequal impact of different surgical procedures on glucose control and suggest that obesity and high pre-operative glucose levels should be considered as risk factors for the development of hyperglycaemia after pancreatic surgery.

Keywords Beta cell mass · Chronic pancreatitis · Diabetes . Insulin secretion · Oral glucose tolerance $\cdot$ Pancreatectomy
Abbreviations
CP chronic pancreatitis
GIP gastric inhibitory polypeptide
GLP-1 glucagon-like peptide-1
MEIA microparticle enzyme immunoassay

\section{Introduction}

Both type 1 and type 2 diabetes have been associated with a significant reduction in beta cell mass, presumably reflecting an increased rate of beta cell apoptosis [1-3]. Thus, diabetes develops when the endocrine pancreas fails to sustain a sufficient amount of insulin secretion for a given degree of insulin resistance. In rodents, hyperglycaemia develops when approximately $80 \%$ of beta cell mass has been lost [4], whereas in larger animals such as pigs and dogs, overt 
diabetes has been observed after a loss of approximately $50 \%$ of beta cells $[5,6]$. Consistent with these animal data, recent studies using pancreas samples from human autopsies have provided evidence that glucose levels begin to rise when the extent of beta cells has been reduced by approximately $50 \%$ [7]; the extent of beta cell loss in patients with overt diabetes is reported to be approximately $65 \%$ [1].

The metabolic consequences arising from such selective beta cell loss are multifaceted and comprise an impairment in glucose-induced insulin secretion and, in particular, a diminished insulin pulse mass, as well as an impairment in peripheral insulin action $[5,6,8]$. However, while a number of previous studies have examined the metabolic changes arising from an experimental reduction of beta cell mass in animal models $[5,6,8,9]$, little is known about the effects of an approximately $50 \%$ partial pancreatectomy in humans [10]. This question is particularly relevant because the capacity for islet regeneration after partial pancreatectomy differs substantially between different species [4, 11-13], thereby potentially leading to marked differences in the insulin secretory capacity following such surgical intervention.

In clinical practice, partial pancreatectomies are commonly performed in patients with pancreatic tumours and tumours of the ampulla of Vater, as well as in patients with chronic pancreatitis suffering from intractable abdominal pain [14]. Indeed, previous studies have alluded to significant impairments in insulin secretion and modest deteriorations in fasting glucose levels in patients undergoing partial pancreatectomy [10, 15-17]. However, the impact of a $50 \%$ partial pancreatectomy on post-challenge glucose excursions has been less well studied. Furthermore, it is unclear whether glucose control is differentially affected by a partial pancreatectomy in patients with different pancreatic diseases [16].

Another interesting question is whether the changes in glucose tolerance and islet function differ between surgical procedures. Thus, while diseases affecting the pancreatic tail are typically treated by distal pancreatectomy, patients with chronic inflammation or tumour formation in the pancreatic head are usually recommended to have proximal pancreaticoduodenectomy (Whipple's operation) with or without pylorus preservation $[16,17]$.

Therefore, in the present study we addressed the following questions: (1) what are the consequences of a $50 \%$ partial pancreatectomy for glucose tolerance and insulin secretion in patients with chronic pancreatitis, patients with pancreatic cancer and patients without diseases affecting pancreatic function; (2) are the alterations in glucose homeostasis and islet-cell secretion induced by a $50 \%$ partial pancreatectomy dependent on the respective surgical procedure (proximal vs distal pancreatectomy); and (3) are any pre-operative predictors of glucose deterioration induced by partial pancreatectomy?

\section{Methods}

Study design Fourteen patients with chronic pancreatitis, ten patients with pancreatic cancer and 13 patients with benign pancreatic tumours or extra-pancreatic masses necessitating partial pancreatectomy were studied. All patients were examined before and after pancreatic surgery with a $240 \mathrm{~min}$ oral glucose challenge, and the respective changes in the plasma concentration profiles of glucose, insulin, C-peptide and non-esterified fatty acids were determined. The study protocol was approved by the ethics committee of the Ruhr-University Bochum (registration number 2528). All patients provided written informed consent prior to study enrolment.

Patients A total of 37 patients (19 men, 18 women) undergoing pancreatic resection in the Department of Surgery, St Josef-Hospital, Ruhr-University Bochum, between the years 2004 and 2007 were included.

The patients were subdivided into three groups: (1) patients with chronic pancreatitis $(n=14)$; (2) patients with pancreatic carcinoma $(n=10)$; and (3) patients with benign pancreatic tumours or extra-pancreatic masses necessitating partial pancreatectomy $(n=13)$.

The group of patients with chronic pancreatitis comprised three female and 11 male patients who underwent pancreatic surgery for the treatment of intractable pain or chronic pancreatic-duct obstruction. Three patients in this group (21\%) had a history of diabetes (treated with glimepiride, diet and exercise, and insulin, respectively). The clinical presentation of these patients suggested the presence of type 2 diabetes, although diabetes secondary to chronic pancreatitis appeared likely given the underlying pancreatic pathology. In addition, five patients had a history of hypertension and three patients had previously been diagnosed with hyperlipidaemia. Three patients had previously undergone appendectomy, and cholecystectomy had been performed in one patient. Twelve patients were current smokers, one patient had a history of nicotine consumption and one patient had never smoked before. Analgesic medication included opioids in two patients $(14 \%)$, and metamizol in three patients (21\%). Two patients (14\%) were taking antidepressant medication and one patient was taking a benzodiazepine. Furthermore, seven patients $(50 \%)$ were taking proton-pump inhibitors and five patients $(36 \%)$ were using antihypertensive medication. One patient (7\%) underwent pancreatic-tail resection, whereas the pancreatic head was removed in the other 13 patients. Amongst the latter group, eight patients were treated by duodenopancreatectomy with pylorus preservation, and five patients underwent duodenum-preserving pancreatic-head resection.

There were six female and four male patients in the group who underwent pancreatic surgery for the removal of 
pancreatic adenocarcinoma. One patient in this group $(10 \%)$ had a history of diabetes (treated with diet and exercise), four patients had a history of arterial hypertension, and hyperlipidaemia was present in five patients. Two patients had previously undergone cholecystectomy, appendectomy had been performed in one case and lower intestinal resections in two cases. One patient was a current smoker, two patients had a history of nicotine consumption and seven patients had never smoked before. Analgesic medication included opioids in one patient $(10 \%)$ and metamizol in two patients $(20 \%)$. One patient $(10 \%)$ was taking antidepressant medication. Four patients $(40 \%)$ were taking proton-pump inhibitors and four patients (40\%) were using antihypertensive medication. Four patients (40\%) underwent pancreatictail resection and the pancreatic head was removed in the other six patients (five patients with duodenopancreatectomy with pylorus preservation and one patient with duodenumpreserving pancreatic-head resection).

The group of patients with benign pancreatic tumours or extra-pancreatic masses necessitating partial pancreatectomy comprised nine female and four male patients who underwent pancreatic surgery for the removal of pancreatic adenoma $(n=12)$ or tumour of the papilla of Vater $(n=1)$. These patients served as controls for the subsequent analyses. None of these patients had a history of diabetes. Hypertension was present in eight patients and eight patients had hyperlipidaemia. Previous abdominal surgery included appendectomy in three patients and cholecystectomy in two patients. Five patients were current smokers and eight patients had never smoked before. No patient in this group was treated with opioids, whereas two patients $(15 \%)$ were taking metamizol. One patient $(8 \%)$ was taking antidepressant medication, six patients $(46 \%)$ were taking proton-pump inhibitors and eight patients $(62 \%)$ were using antihypertensive medication. Seven patients $(46 \%)$ underwent pancreatic-tail resection, whereas the pancreatic head was removed in the other six patients (five patients with duodenopancreatectomy with pylorus preservation and one patient with a typical Whipple's operation).

The clinical diagnoses chronic pancreatitis, pancreatic carcinoma, pancreatic adenoma or ampullary cancer were confirmed by an independent pathologist in all cases. Detailed patient characteristics are presented in Table 1.

Experimental procedures The experiments were performed in the morning after an overnight fast with participants in a supine position throughout the experiments. All concomitant medication was withdrawn on the evening of the preceding day. No restrictions were made regarding the intake of water and tobacco use until the morning of the experiments. Both ear lobes were made hyperaemic using Finalgon (Nonivamide $4 \mathrm{mg} / \mathrm{g}$, Nicoboxil $25 \mathrm{mg} / \mathrm{g}$; Boehringer Ingelheim Pharma, Ingelheim, Germany). The experiments were started by the ingestion of the oral glucose load ( $75 \mathrm{~g}$ glucose in $300 \mathrm{ml}$ ) over $5 \mathrm{~min}$, and capillary and venous blood samples were drawn at $t=-5,0,15,30,60,90,120$, 150, 180, 210 and $240 \mathrm{~min}$. Capillary blood samples (approximately $100 \mu \mathrm{l}$ ) were added to $\mathrm{NaF}$ (Microvette CB

Table 1 Clinical characteristics determined before pancreatic surgery in patients with chronic pancreatitis or pancreatic cancer, and in the control group

\begin{tabular}{|c|c|c|c|c|}
\hline Characteristic & Chronic pancreatitis & Pancreatic cancer & Control group & $p$ value $^{\mathrm{a}}$ \\
\hline Age (years) & $53.4 \pm 10.1$ & $68.6 \pm 7.9^{*}$ & $58.8 \pm 15.0$ & 0.012 \\
\hline Sex (women/men) & $3 / 11^{*}$ & $6 / 4$ & $9 / 4$ & 0.019 \\
\hline BMI $\left(\mathrm{kg} / \mathrm{m}^{2}\right)$ & $21.9 \pm 3.5$ & $25.2 \pm 4.5$ & $24.7 \pm 4.5$ & 0.11 \\
\hline Waist:hip ratio & $0.92 \pm 0.02$ & $0.88 \pm 0.02$ & $0.87 \pm 0.03$ & 0.29 \\
\hline RR systolic (mmHg) & $115.7 \pm 16.0$ & $124.5 \pm 17.9$ & $133.5 \pm 20.1$ & 0.054 \\
\hline $\mathrm{RR}$ diastolic $(\mathrm{mmHg})$ & $73.9 \pm 10.0$ & $74.5 \pm 9.0$ & $78.5 \pm 12.1$ & 0.50 \\
\hline $\mathrm{HbA}_{1 \mathrm{c}}(\%)$ & $6.26 \pm 0.59$ & $6.09 \pm 0.55$ & $5.83 \pm 0.97$ & 0.33 \\
\hline White blood count $\left(10^{9} / 1\right)$ & $7.152 \pm 2.048$ & $7.918 \pm 3.136$ & $6.788 \pm 1.493$ & 0.49 \\
\hline Haemoglobin $(g / l)$ & $133 \pm 14$ & $124 \pm 12$ & $137 \pm 18$ & 0.15 \\
\hline Serum amylase (U/l) & $79.5 \pm 120.4$ & $40.2 \pm 38.7$ & $77.1 \pm 141.0$ & 0.66 \\
\hline Serum lipase $(U / 1)$ & $176.0 \pm 280.8$ & $120.0 \pm 134.2$ & $127.3 \pm 338.9$ & 0.87 \\
\hline C-reactive protein $(\mathrm{mg} / \mathrm{l})$ & $71 \pm 90$ & $127 \pm 178$ & $43 \pm 38$ & 0.20 \\
\hline Ca $19-9(\mathrm{kU} / \mathrm{l})$ & $22.7 \pm 44.3$ & $453.9 \pm 1107.4$ & $31.1 \pm 46.5$ & 0.17 \\
\hline $\mathrm{CEA}(\mu \mathrm{g} / \mathrm{l})$ & $2.73 \pm 1.51$ & $4.06 \pm 5.80$ & $1.93 \pm 1.63$ & 0.34 \\
\hline Triacylglycerol (mmol/l) & $1.62 \pm 0.66$ & $1.93 \pm 1.28$ & $1.54 \pm 0.75$ & 0.12 \\
\hline Cholesterol $(\mathrm{mmol} / \mathrm{l})$ & $4.81 \pm 1.02$ & $5.18 \pm 2.0$ & $6.0 \pm 1.24$ & 0.60 \\
\hline
\end{tabular}

Data shown are means \pm SD

${ }^{a}$ ANOVA or $\chi^{2}$ test

${ }^{*} p<0.05$ vs control group (Duncan's post hoc test)

Ca 19-9, carbohydrate antigen 19-9; CEA, carcinoembryonic antigen; RR, Riva Rocci 
300; Sarstedt, Nümbrecht, Germany) for the immediate measurement of glucose. Venous blood was drawn into chilled tubes containing EDTA and aprotinin (Trasylol; 20,000 kIU/ml, $200 \mu \mathrm{l} / 10 \mathrm{ml}$ blood; Bayer AG, Leverkusen, Germany) and kept on ice. After centrifugation at $4^{\circ} \mathrm{C}$, plasma for hormone analyses was kept frozen at $-28^{\circ} \mathrm{C}$.

The time interval between the first oral glucose challenge and the date of surgery was $8.0 \pm 7.6$ days (mean $\pm \mathrm{SD}$; range: 1-36 days), and the second test was carried out $23.9 \pm$ 27.0 days after surgery (range: 4-106 days).

Measurements Glucose was measured as described [18, 19] using a glucose oxidase method with a Glucose Analyzer 2 (Beckman Instruments, Munich, Germany).

Insulin was measured as described [18] using an insulin microparticle enzyme immunoassay (MEIA), (IMx Insulin, Abbott Laboratories, Wiesbaden, Germany). Cross-reactivity with proinsulin was $<0.005 \%$. The intra-assay coefficient of variation was $4 \%$.

C-peptide was measured as described [18] using an ELISA from DAKO (Cambridge, UK). The intra-assay coefficient of variation was $3.3-5.7 \%$ and the inter-assay variation was $4.6-5.7 \%$. Human insulin and C-peptide were used as standards.

NEFA were determined by spectrophotometric analysis as described [18], using reagents from Wako Chemicals (Neuss, Germany).

Calculations The Matsuda index of insulin sensitivity was calculated as described [20, 21]. Integrated incremental plasma concentrations of insulin and C-peptide were calculated according to the trapezoidal rule (baseline subtracted).

Statistical analysis Participant characteristics are reported as mean $\pm \mathrm{SD}$ and results are presented as mean $\pm \mathrm{SEM}$. Time-course measurements were analysed by paired or unpaired ANOVA, as appropriate, using Statistica version 5.0 (Statsoft Europe, Hamburg, Germany). Three different $p$ values were calculated: (1) for the overall differences between the different groups/experiments, independent of the respective time patterns; (2) for the differences over the time course, independent of the respective groups/experiments; and (3) for the determination of differences between the groups/experiments over the time course. If a significant $(p<0.05)$ interaction between group/experiment and time was documented, values at single time points were compared by one-way ANOVA or Student's $t$ test. All other variables were compared by one-way ANOVA or Student's $t$ test, as appropriate. A $p$ value $<0.05$ was taken to indicate a significant difference. Regression analyses were carried out using GraphPad Prism 4 (San Diego, CA, USA).

\section{Results}

Comparison of pre-operative metabolic variables between the groups Fasting glucose concentrations were $6.48 \pm$ $0.37 \mathrm{mmol} / 1$ in patients with chronic pancreatitis, $5.92 \pm$ $0.35 \mathrm{mmol} / \mathrm{l}$ in patients with pancreatic carcinoma, and $5.65 \pm 0.38 \mathrm{mmol} / \mathrm{l}$ in control participants ( $p=0.26$; Table 2$)$. Oral glucose ingestion elicited a significant rise in glycaemia in all three groups $(p<0.0001$ for the time course; Fig. 1). The post-challenge glucose excursions were significantly higher in patients with chronic pancreatitis and with pancreatic carcinoma than in control participants ( $p=0.0032$ for the interaction of group and time course).

The rise in plasma glucose concentration was accompanied by a marked increase in insulin and C-peptide levels in all three groups ( $p<0.001$ for the time course). The absolute plasma concentrations of insulin and C-peptide reached after oral glucose ingestion were highest in the control group,
Table 2 Metabolic variables determined before and after pancreatic surgery in patients with chronic pancreatitis or pancreatic cancer, and in the control group
Data are means \pm SD

a Paired Student's $t$ test

\begin{tabular}{lccc}
\hline Variable & Before surgery & After surgery & $p$ value $^{\mathrm{a}}$ \\
\hline Chronic pancreatitis & & & \\
Fasting glucose $(\mathrm{mmol} / \mathrm{l})$ & $6.47 \pm 0.39$ & $6.87 \pm 0.44$ & 0.34 \\
$\mathrm{AUC}_{\text {insulin }}(\mathrm{pmol} / \mathrm{l} \times \mathrm{min})$ & $670.2 \pm 103.7$ & $341 \pm 51.8$ & 0.0021 \\
$\mathrm{AUC}_{\mathrm{C} \text {-peptide }}(\mathrm{nmol} / \mathrm{l} \times \mathrm{min})$ & $291.4 \pm 35.1$ & $195 \pm 28.5$ & 0.0065 \\
Matsuda index & $9.25 \pm 1.13$ & $11.05 \pm 0.62$ & 0.12 \\
Pancreatic cancer & & & \\
Fasting glucose $(\mathrm{mmol} / \mathrm{l})$ & $5.92 \pm 0.33$ & $6.7 \pm 0.33$ & 0.17 \\
AUC $_{\text {insulin }}(\mathrm{pmol} / \mathrm{l} \times \mathrm{min})$ & $1,077.5 \pm 186.7$ & $515 \pm 75.2$ & 0.0159 \\
$\mathrm{AUC}_{\mathrm{C}-\text { peptide }}(\mathrm{nmol} / \mathrm{l} \times \mathrm{min})$ & $412.9 \pm 59.9$ & $257.9 \pm 286.9$ & 0.066 \\
Matsuda index $_{\text {Control group }}$ & $5.14 \pm 0.71$ & $7.59 \pm 1.25$ & 0.087 \\
Fasting glucose $(\mathrm{mmol} / \mathrm{l})_{\mathrm{AUC}_{\text {insulin }}(\mathrm{pmol} / \mathrm{l} \times \mathrm{min})}$ & $5.64 \pm 0.39$ & $6.53 \pm 0.39$ & 0.041 \\
AUC $_{\mathrm{C} \text {-peptide }}(\mathrm{nmol} / \mathrm{l} \times \mathrm{min})$ & $1476.7 \pm 262.3$ & $646.5 \pm 87$ & 0.0083 \\
Matsuda index & $486.4 \pm 75.2$ & $314.9 \pm 30.5$ & 0.0503 \\
\hline
\end{tabular}



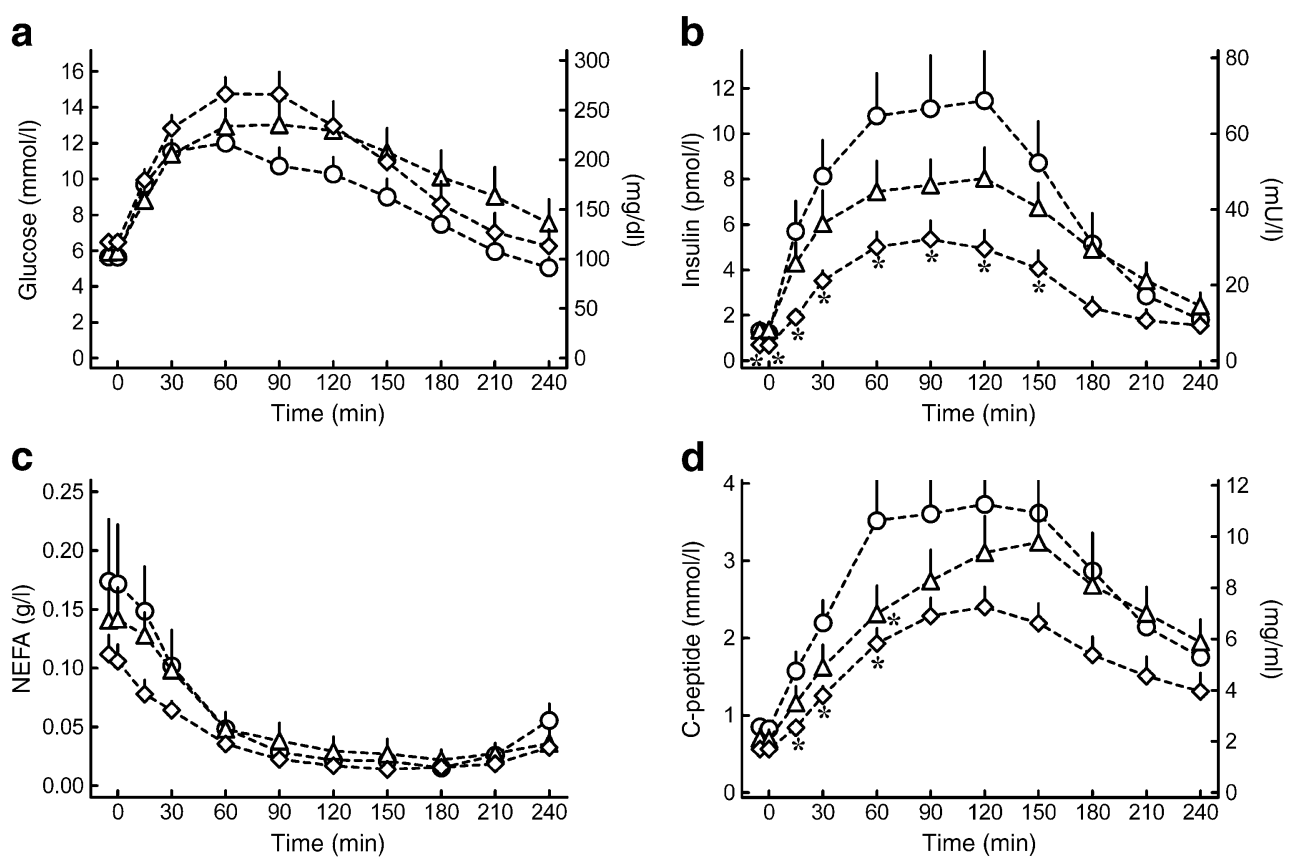

Fig. 1 Plasma concentrations of glucose (a), insulin (b), NEFA (c) and C-peptide (d) in 14 patients with chronic pancreatitis (diamonds), ten patients with pancreatic carcinoma (triangles) and 13 control participants (circles) examined prior to pancreatic surgery. Data are presented as means \pm SEM. Statistical analyses were carried out using unpaired repeated-measures ANOVA and denote: (1) overall differences between the three groups, glucose $p=0.31$, insulin $p=0.010$,

and lowest in patients with chronic pancreatitis $(p=0.0027$ and $p=0.0089$ for the interaction of group and time course, respectively; Fig. 1). Thus, the incremental integrated insulin concentrations were $670.2 \pm 103.7 \mathrm{pmol} \mathrm{l}^{-1} \mathrm{~min}$ in chronic pancreatitis, $1,077.5 \pm 186.7 \mathrm{pmol}^{-1} \mathrm{~min}$ in patients with pancreatic carcinoma, and $1,476.7 \pm 262.3 \mathrm{pmol}^{-1} \mathrm{~min}$ in controls $(p=0.015$; Table 2).

NEFA levels were significantly lowered by the oral glucose load ( $p<0.0001$ for the time course), but there were no differences in NEFA levels between the groups $(p=0.52$ for the interaction of group and time course; Fig. 1).

The Matsuda index for insulin sensitivity was $9.25 \pm 1.13$ in patients with chronic pancreatitis, $5.14 \pm 0.71$ in patients with pancreatic cancer and $5.59 \pm 0.73$ in controls $(p=0.0054)$.

Metabolic changes after partial pancreatectomy In the control group, fasting glucose concentrations increased significantly after partial pancreatectomy $(p=0.041$; Table 2). While the initial rise in glucose concentrations after the glucose load $(t=30-90 \mathrm{~min})$ was slightly shallower than in the experiments before the partial pancreatectomy, the glucose levels at $t=180-240$ were significantly higher than before surgery $(p<0.0001$ for the interaction of experiment and time course; Fig. 2). Postchallenge insulin and C-peptide excursions were markedly lowered by the partial pancreatectomy (Fig. 2; Table 2).

NEFA $p=0.25$, C-peptide $p=0.032 ;$ (2) differences over the time course, glucose $p<0.0001$, insulin $p<0.0001$, NEFA $p<0.0001$, C-peptide $p<0.0001$; and (3) differences between the groups over the time course, glucose $p=0.0032$, insulin $p=0.0027$, NEFA $p=0.52$, C-peptide $p=0.0089$. Significant differences $(* p<0.05)$ at individual time points vs controls (one-way ANOVA)

NEFA levels were not significantly changed after the partial pancreatectomy ( $p=0.99$ for the interaction between experiment and time course; Fig. 2). The Matsuda index for insulin sensitivity was similar before and after surgery (Table 2).

In patients with chronic pancreatitis (CP), fasting glucose concentrations were unchanged by the partial pancreatectomy (Fig. 3). However, glucose concentrations between $t=30 \mathrm{~min}$ and $t=90 \mathrm{~min}$ were significantly lowered by the procedure, whereas higher glucose levels were reached between $t=180 \mathrm{~min}$ and $t=240 \mathrm{~min}$ in the experiments carried out after surgery $(p<0.0001$ for the interaction of experiment and time course; Fig. 3). Both insulin and C-peptide levels were significantly lowered by the partial pancreatectomy (Fig. 3; Table 2). Following surgery, NEFA levels were significantly higher in the fasting state as well as until $180 \mathrm{~min}$ after oral glucose ingestion ( $p=0.002$ for the interaction of experiment and time; Fig. 3). There were no changes in the Matsuda index after the partial pancreatectomy (Table 2).

In patients with pancreatic cancer, the overall patterns of post-challenge glucose concentrations were characterised by a slightly smoother initial increment after glucose ingestion $(t=30-120 \mathrm{~min})$, and higher levels at the subsequent time points $(p=0.0005$ for the interaction of group and time; Fig. 4). As seen in the other two groups, post-challenge 
a
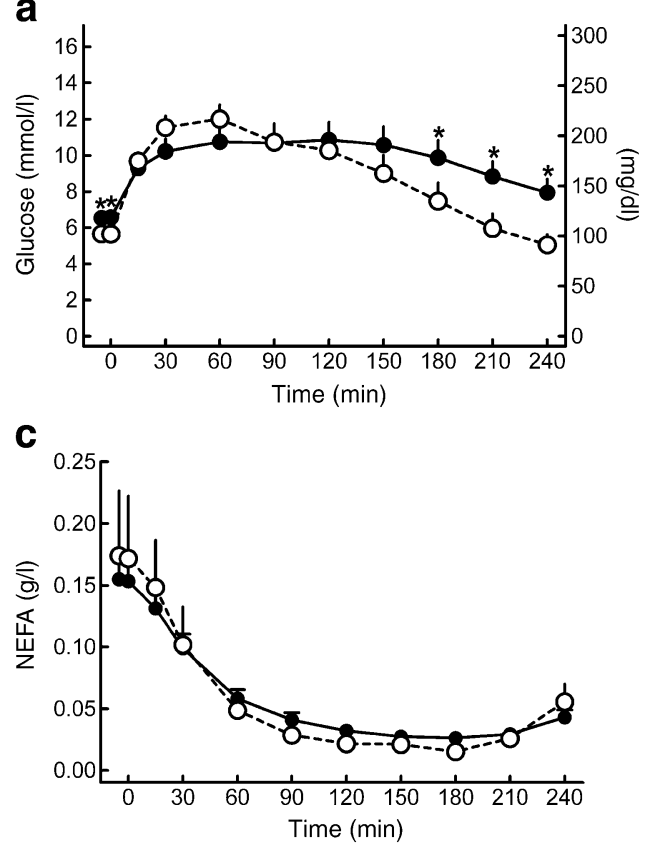

b

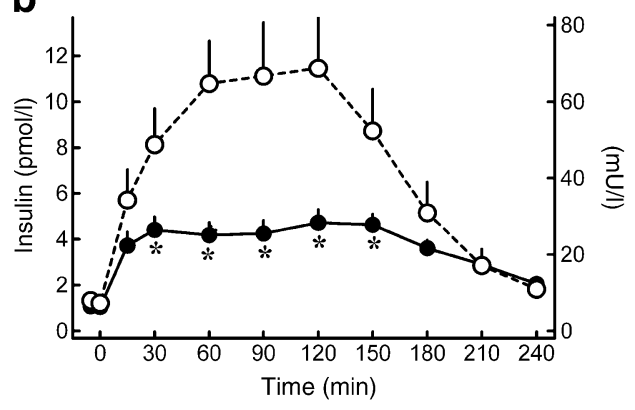

d

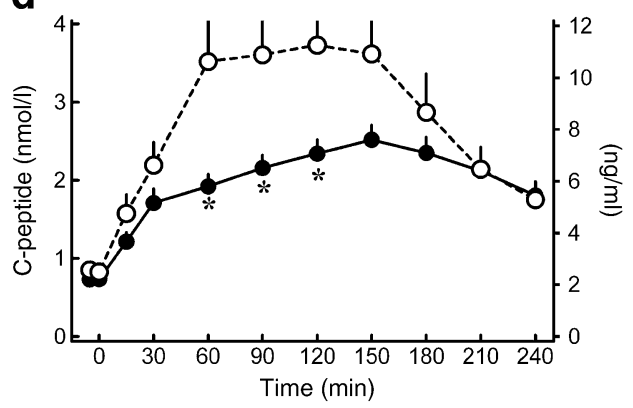

carried out before and after surgery, glucose $p=0.25$, insulin $p=0.008$, NEFA $p=0.96$, C-peptide $p=0.024$; (2) differences over the time course, glucose $p<0.0001$, insulin $p<0.0001$, NEFA $p<0.0001$, C-peptide $p<0.0001$; and (3) differences between the two experiments over the time course, glucose $p<0.0001$, insulin $p<0.0001$, NEFA $p=0.99$, C-peptide $p<0.0001$. Significant differences $\left({ }^{*} p<0.05\right)$ at individual time points (one-way ANOVA)

b
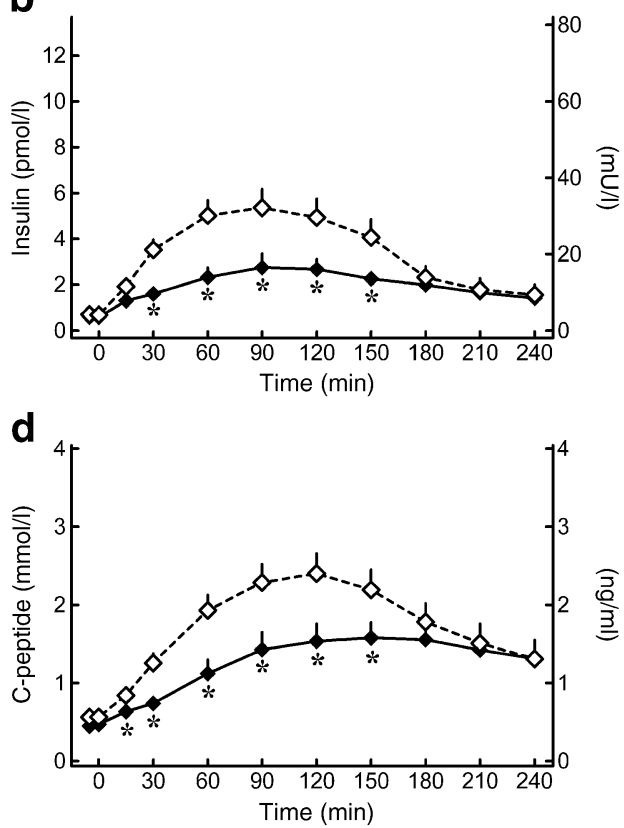

C-peptide $p=0.01$; (2) differences over the time course, glucose $p<0.0001$, insulin $p<0.0001$, NEFA $p<0.0001$, C-peptide $p<0.0001$; and (3) differences between the two experiments over the time course, glucose $p<0.0001$, insulin $p<0.0001$, NEFA $p=0.002$, C-peptide $p<0.0001$. Significant differences $\left({ }^{*} p<0.05\right)$ at individual time points (one-way ANOVA) (1) overall differences between experiments carried out before and after surgery, glucose $p=0.91$, insulin $p=0.0054$, NEFA $p=0.0032$, 

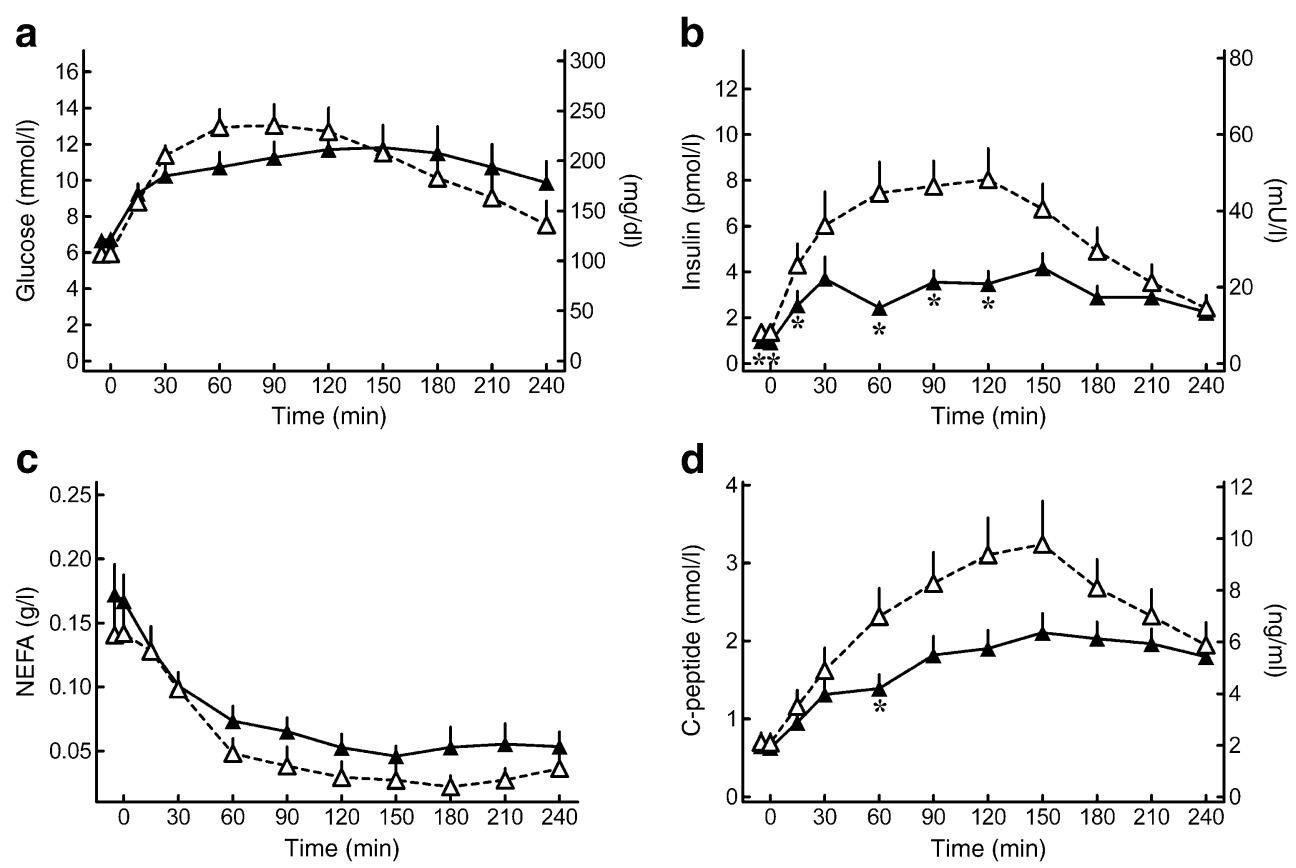

Fig. 4 Plasma concentrations of glucose (a), insulin (b), NEFA (c) and C-peptide (d) in ten patients with pancreatic carcinoma studied before (white triangles) and after pancreatic surgery (black triangles). Data are presented as means \pm SEM. Statistical analyses were carried out using unpaired repeated-measures ANOVA and denote: (1) overall differences between experiments carried out before and after surgery,

glucose $p=0.90$, insulin $p=0.0061$, NEFA $p=0.025$, C-peptide $p=0.09$; (2) differences over the time course, glucose $p<0.0001$, insulin $p<0.0001$, NEFA $p<0.0001$, C-peptide $p<0.0001$; and (3) differences between the two experiments over the time course, glucose $p=0.0005$, insulin $p<0.0001$, NEFA $p=0.80$, C-peptide $p=0.0007$. Significant differences $\left({ }^{*} p<0.05\right)$ at individual time points (one-way ANOVA)

insulin and C-peptide levels were lowered by the surgical intervention (Fig. 4; Table 2). There were no significant differences in NEFA levels between the experiments before and after pancreatic surgery ( $p=0.80$ for the interaction of experiment and time). Insulin sensitivity was not significantly improved after the partial pancreatectomy ( $p=0.087$; Table 2$)$.

Dependency of metabolic changes on the surgical procedure In order to examine the dependency of the changes in glycaemia and insulin secretion on the respective surgical procedure, the control group was subdivided into patients undergoing pancreatic-head resection $(n=6)$ and those undergoing pancreatic-tail resection $(n=7)$. There was a similar reduction of post-challenge excursions in insulin and C-peptide levels in patients undergoing pancreatic-head and -tail resections (Fig. 5). Incremental insulin levels after the oral glucose load were $1,206.8 \pm 333.2 \mathrm{pmol} \mathrm{min}^{-1} \mathrm{~min}$ before and $500.5 \pm 94.8 \mathrm{pmol} \mathrm{min}^{-1} \mathrm{~min}$ after pancreatic-head resection (58\% reduction), and $1,711.5 \pm 393.3 \mathrm{pmol} \mathrm{min}^{-1} \mathrm{~min}$ before and $802.5 \pm 121.2 \mathrm{pmol} \mathrm{min}^{-1} \mathrm{~min}$ after pancreatictail resection (53\% reduction). However, in patients undergoing pancreatic-head resection, the initial rise in glycaemia between $t=30$ and $t=60 \mathrm{~min}$ after the oral glucose load was even lower after surgery, whereas glucose levels between $t=180$ and $t=240 \mathrm{~min}$ tended to be even higher (Fig. 5; $p<0.0001$ for the interaction of experiment

and time). In contrast, glucose concentrations tended to be higher at all time points after pancreatic-tail resection ( $p=0.041$ for the interaction of experiment and time).

Predictors of deterioration in glycaemic control after partial pancreatectomy In order to identify pre-operative predictors of poor glycaemic control after a $50 \%$ partial pancreatectomy, the post-operative fasting glucose concentrations were correlated to various anthropometric variables and pre-operative measures of glucose control. There was a significantly positive association between pre- and postoperative fasting glucose levels (Fig. 6). Similarly, high pre-operative values for body weight, BMI and waist circumference were correlated with post-operative glucose concentrations (Fig. 6). No relationship was detected between age, incremental integrated insulin levels after oral glucose ingestion or the Matsuda insulin sensitivity index and the respective fasting glucose levels after partial pancreatectomy (details not shown).

\section{Discussion}

The present study was designed to examine the metabolic changes after a $50 \%$ partial pancreatectomy in humans. We report that: (1) insulin secretion was reduced by approxi- 
a

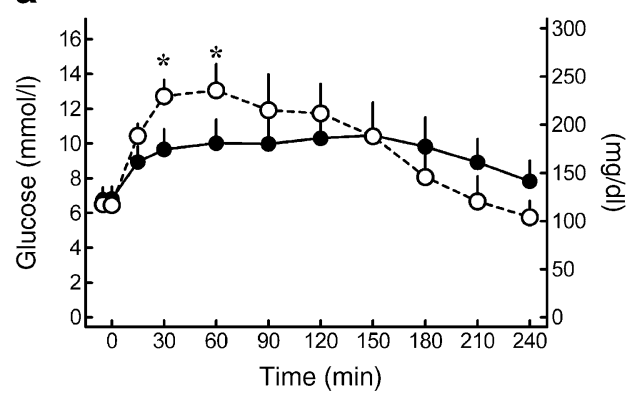

C

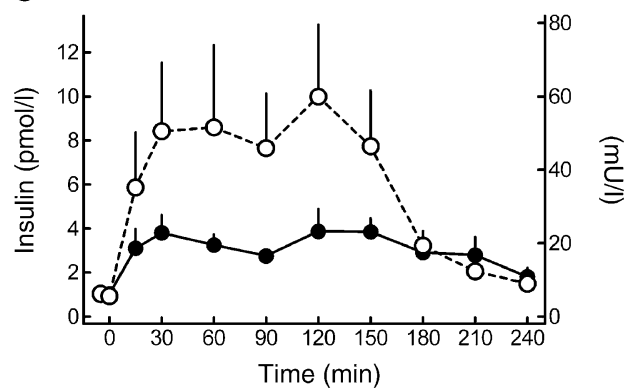

e

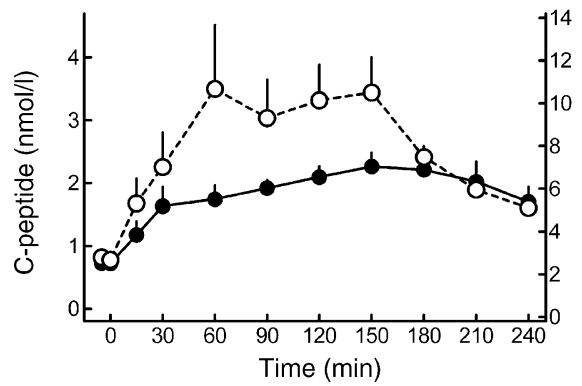

Fig. 5 Plasma concentrations of glucose (a, b), insulin (c, d), and C-peptide (e, f) in 13 patients undergoing pancreatic-head (a, c, e; $n=6)$ or -tail $(\mathbf{b}, \mathbf{d}, \mathbf{f} ; n=7)$ resection for the removal of benign tumours or extrapancreatic masses, studied before (white circles) and after pancreatic surgery (black circles). Data are presented as means \pm SEM. Statistical analyses were carried out using unpaired repeatedmeasures ANOVA and denote: (1) overall differences between experiments carried out before and after surgery: pancreatic-head resection, glucose $p=0.68$, insulin $p=0.061$, C-peptide $p=0.016$;

mately $50 \%$ after partial pancreatectomy in patients with chronic pancreatitis, patients with pancreatic cancer and controls; (2) in the control group, surgical removal of the pancreatic tail led to deteriorations in fasting and postchallenge glycaemia, whereas pancreatic-head resection caused a transient reduction in glycaemic excursions even immediately following oral glucose ingestion; (3) high preoperative body weight and elevated fasting glucose levels were associated with poor glycaemic control after partial pancreatectomy.

The transient improvements in glycaemia after partial pancreatectomy observed in all three groups immediately after the oral glucose load despite the approximately $50 \%$ reduction in insulin concentrations are rather surprising. b

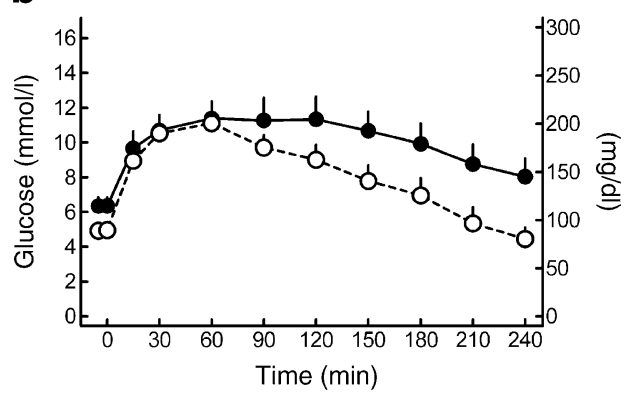

d

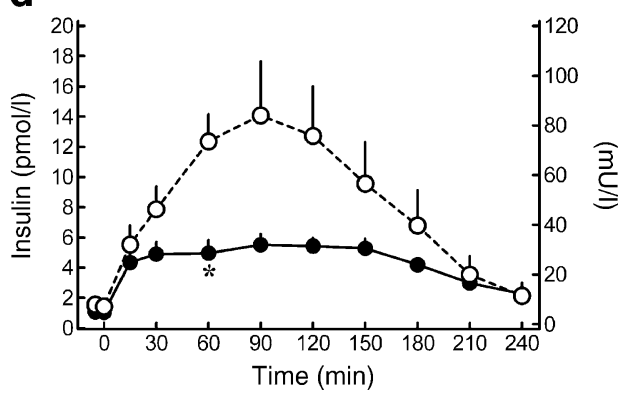

f

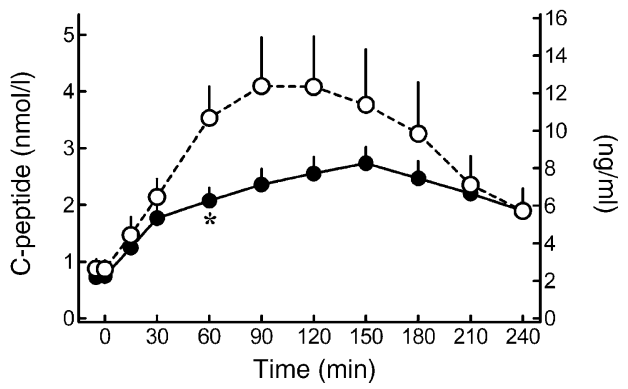

pancreatic-tail resection, glucose $p=0.094$, insulin $p=0.077$, C-peptide $p=0.19$ (2) differences over the time course: pancreatic-head and -tail resections, glucose $p<0.0001$, insulin $p<0.0001$, NEFA $p<0.0001$, C-peptide $p<0.0001$, for both procedures; and (3) differences between the two experiments over the time course, pancreatic-head resection, glucose $p<0.0001$, insulin $p=0.011$, C-peptide $p=0.055$; pancreatictail resection, glucose $p=0.041$, insulin $p=0.0008$, C-peptide $p=0.039$. Significant differences $\left({ }^{*} p<0.05\right)$ at individual time points (one-way ANOVA)

Indeed, any reduction in beta cell mass and insulin secretion would be expected to result in a worsening of post-challenge glucose control $[3,5,8]$. Therefore, the question arises what mechanisms were responsible for the reduction in glycaemic excursions observed in these patients; two possibilities come to mind. First, it is possible that local inflammatory signals in patients with chronic pancreatitis and the release of cytokines from pancreatic tumours were partly responsible for the higher glucose excursions prior to surgery, and that removal of these tissue areas caused the improvements in glucose homeostasis observed in this study $[22,23]$. This argument implies that the alterations in glucose tolerance found in some patients with chronic pancreatitis or pancreatic tumours prior to surgery were primarily caused by defects in 


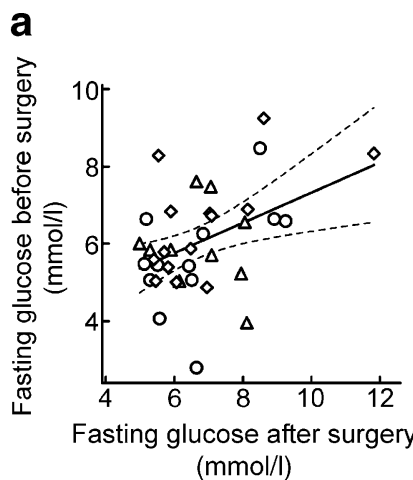

b
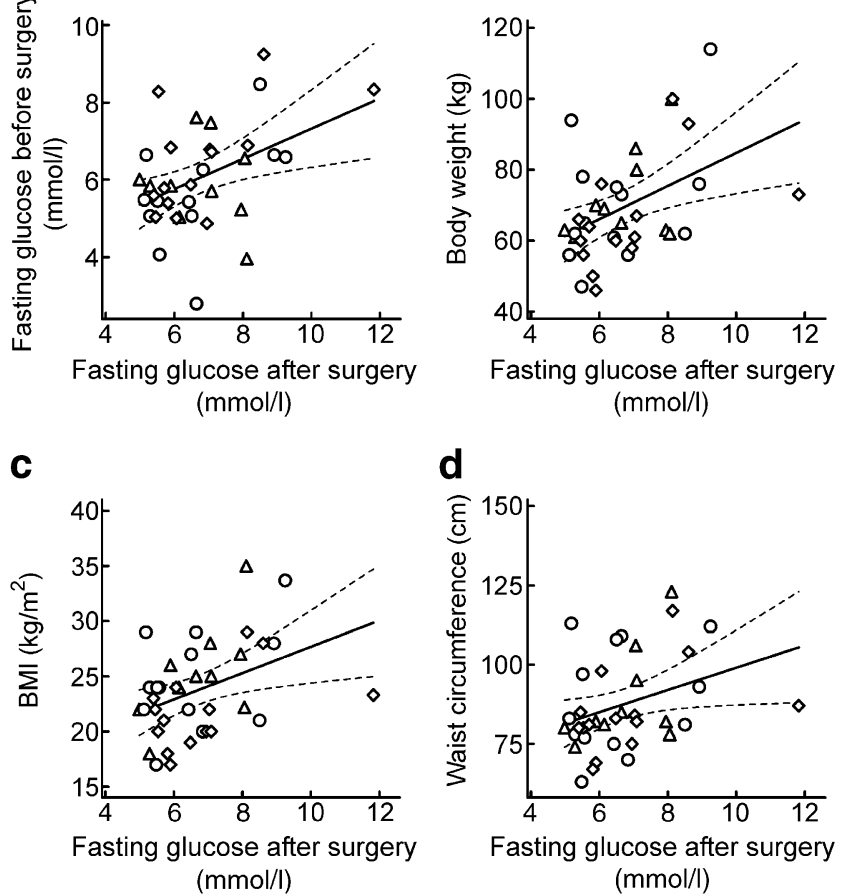

d

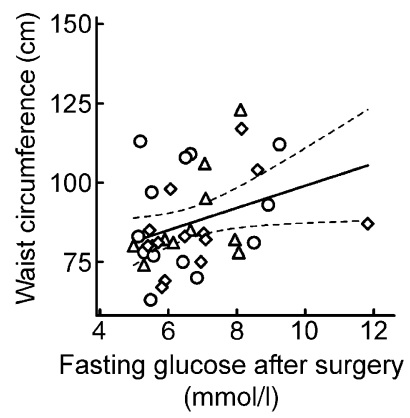

Fig. 6 Linear regression analysis of the post-operative fasting glucose concentration and (a) the pre-operative glucose level, $p=0.0073$, bivariate correlation coefficient $(r)=0.43$, (b) the pre-operative body weight, $p=0.0056, r=0.45$, (c) the pre-operative body mass index, $p=0.012, r=0.41$, and (d) the pre-operative waist circumference, $p=0.037, r=0.34$, in 14 patients with chronic pancreatitis (diamonds), ten patients with pancreatic carcinoma (triangles) and 13 controls (circles). The solid line indicates the regression line, dashed lines indicate the respective upper and lower 95\% confidence intervals

insulin action that were restored after partial pancreatectomy [24]. However, the fact that insulin sensitivity was not significantly improved after partial pancreatectomy argues against such reasoning.

It is more likely that the transient reductions in postchallenge glucose excursions were caused by a delay in gastric emptying induced by the surgical intervention. Indeed, the rate of gastric emptying is known to be one of the major predictors of postprandial glycaemia, accounting for approximately $35 \%$ of the total variation in postprandial glucose level $[25,26]$, and decelerating the velocity of gastric emptying is commonly applied as strategy in the treatment of patients with type 2 diabetes using, for example, glucagon-like peptide-1 (GLP-1) analogues or amylin [27, 28]. Such alterations in stomach motility induced by proximal pancreatectomy would also explain why glucose levels were lowered only within the first $120 \mathrm{~min}$ after oral glucose ingestion, increasing at the subsequent time points. In this regard, it is noteworthy that reductions in postchallenge glycaemia were observed only in patients undergoing pancreatic-head resections, whereas surgical removal of the pancreatic tail led to deteriorations in glucose control at all time points during the oral glucose tolerance test. This is consistent with previous studies showing a significantly higher incidence of delayed gastric emptying after proximal pancreatectomy than after distal pancreatectomy [14, 29-31]. Thus, the delay in gastric emptying observed in some patients after pancreatic-head resection appears to provide a safeguard against the development of postprandial hyperglycaemia. In contrast, removing the pancreatic tail seems to be associated with an increased risk of developing overt hyperglycaemia.

Another factor that might theoretically worsen postprandial glucose control, especially after duodenopancreatectomy, is the removal of parts of the small intestine. Indeed, as the intestine also contains gastric inhibitory polypeptide (GIP)and GLP-1-secreting $\mathrm{K}$ and L cells, it is theoretically possible that removing parts of the lower intestine would also impair the secretion of incretin hormones and thus diminish postprandial insulin secretion. In line with such reasoning, previous studies have reported small reductions in the level of GIP, but not GLP-1, after duodenopancreatectomy [32, 33]. However, as the majority of $\mathrm{L}$ cells are located in lower parts of the intestine, and given the rather small amount of intestinal tissue removed from patients in the present study, the overall impact of such disturbances in the enteroinsular axis should be relatively minor.

In the present study, patients with chronic pancreatitis already exhibited significant impairments in insulin secretion before surgery, and this was associated with higher glycaemic excursions following the oral glucose load. This finding is consistent with previous studies and probably reflects the destruction of islet beta cells in such patients $[34,35]$. In contrast, insulin sensitivity was even greater in patients with chronic pancreatitis compared with the other groups, most likely as a consequence of intestinal malnutrition and an overall lower BMI in these patients.

Although insulin secretion was diminished by approximately $50 \%$ in all three groups of patients, NEFA concentrations were increased after surgery only in patients with chronic pancreatitis. This is most likely to be due to the fact that pre-operative insulin levels were already significantly lower in the CP patients than in the other groups, meaning that the additional reduction in insulin secretion induced by the partial pancreatectomy had further diminished the insulin secretory capacity in these patients below a critical extent required to suppress lipolysis adequately. In contrast, the residual insulin secretion in the other two groups may have still been sufficient to elicit a normal suppression of NEFA release.

Significant improvements in glucose tolerance following partial pancreatectomy have previously been reported in patients with pancreatic cancer $[15,36]$. This finding has been attributed to an improvement in insulin sensitivity after removal of the pancreatic tumour [36]. The present data are partly consistent with these studies as they show 
improvement in glucose tolerance following the partial pancreatectomy. However, the lack of significant changes in insulin resistance, the fact that a similar reduction in post-challenge glucose excursion was also found in patients with non-malignant pancreatic disease and the significant increase in glucose level 3-4 h after the oral glucose load suggest that this phenomenon is rather non-specific for pancreatic cancer and is likely to be mediated by a delay in gastric emptying.

A number of previous reports, predominantly based on rodent studies, have suggested that the majority of beta cells are located in the pancreatic tail, whereas the extent of beta cells in the pancreatic head is often held to be negligible [37, 38]. This finding has often been used as a rationale for removing the pancreatic head in patients with chronic pancreatitis. In contrast, recent studies have demonstrated that, unlike in rodents, the distribution of islets in humans is rather homogeneous throughout the pancreas, with minimal differences in the respective beta cell content between the pancreatic head and tail only [39]. The present data lend support to these studies by showing that the quantitative impairment in post-challenge insulin secretion is rather similar in patients undergoing proximal and distal pancreatectomy. Thus, while the distribution of beta cells may vary throughout the pancreas in rodents, these differences appear to be rather negligible in humans.

A recent study by Matveyenko and colleagues has examined the effects of a $50 \%$ pancreatectomy on insulin secretion and insulin sensitivity in dogs [5]. Under experimental conditions, the surgical reduction of beta cell mass was associated with a concomitant deterioration in both insulin secretion and peripheral insulin sensitivity. The present data seem to be at variance with this study by showing no changes in insulin sensitivity following the surgical intervention. However, by definition the present study included patients with pancreatic disease only, whereas healthy animals were examined in the dog study. As both chronic inflammation and tumour-induced cytokine release may alter peripheral insulin action [22], it is possible that the improvements in insulin sensitivity seen in the present study were secondary to the removal of these pathological pancreatic areas, and that a similar 50\% partial pancreatectomy in completely healthy humans would have had a different impact on insulin action.

Some limitations may apply to the present results. Although the patterns of post-challenge glucose excursions after pancreatic-head resection suggest a delay in gastric emptying, this has not been directly assessed in the present study. However, previous studies have provided evidence that gastric emptying is delayed in a significant percentage of patients undergoing pancreaticoduodenectomy with pylorus preservation, whereas the incidence of delayed gastric emptying has been reported to be relatively low in patients treated with a distal pancreatectomy [14, 29-31]. Furthermore, while the average extent of pancreatic resection has been estimated as 50\% for all the procedures applied in this study, the individual percentage may have varied between 40 and $60 \%$. However, as the exact weight of the pancreas cannot readily be determined in vivo during surgery, this limitation is unavoidable in human studies such as this.

What are the clinical consequences of this study? Indeed, the modest improvements in glucose tolerance after proximal pancreaticoduodenectomy may be interpreted as an argument in favour of a surgical approach for the treatment of patients with chronic pancreatitis. It is, however, important to note that this study has evaluated only the immediate consequences of a partial pancreatectomy and that the long-term results of this intervention may be different. In line with this, previous studies in healthy humans who donated $50 \%$ of their pancreas to relatives with type 1 diabetes have revealed an increased risk of diabetes in the donors [10, 40, 41], and previous long-term follow-up studies in patients with chronic pancreatitis have alluded to a progressive increase in the incidence of diabetes subsequent to a partial pancreatectomy [16, 42-44]. The present studies have also identified high pre-operative BMI and fasting glucose levels as risk factors for post-operative deteriorations in glucose control. Thus, the risk of worsening post-operative glucose control should be critically borne in mind when subjecting obese patients with elevated preoperative glucose levels to a partial pancreatectomy.

In conclusion, the present study has shown that insulin secretion is diminished by approximately 50\% after both proximal and distal pancreatectomy. However, despite lower insulin responses to an oral glucose load, post-challenge glucose tolerance was ameliorated after pancreatic-head resection, whereas removal of the pancreatic tail was associated with significant deteriorations of post-challenge glycaemia. The risk of inducing post-operative deterioration in glucose control was highest in obese patients with elevated fasting glucose levels. These data highlight the unequal impact of different surgical procedures on post-operative glucose control and suggest that obesity and high preoperative glucose levels should be taken into consideration as potential risk factors for the post-operative development of hyperglycaemia.

Acknowledgements The excellent technical assistance of B. Baller, K. Mros, H. Achner and G. Müller is gratefully acknowledged. These studies were supported by grants from the Deutsche Forschungsgemeinschaft (DFG grant-no. Me2096/5-1 to J. J. Meier) and the Ruhr-University of Bochum (FoRUM grants to J. J. Meier).

Duality of interest The authors declare that there is no duality of interest associated with this manuscript. 


\section{References}

1. Butler AE, Janson J, Bonner-Weir S, Ritzel R, Rizza RA, Butler PC (2003) Beta-cell deficit and increased beta-cell apoptosis in humans with type 2 diabetes. Diabetes 52:102-110

2. Meier JJ, Bhushan A, Butler AE, Rizza RA, Butler PC (2005) Sustained beta-cell apoptosis in patients with long-standing type 1 diabetes: Indirect evidence for islet regeneration. Diabetologia 48:2221-2228

3. Meier JJ (2008) Beta cell mass in diabetes: a realistic therapeutic target. Diabetologia 51:703-713

4. Leahy JL, Bonner-Weir S, Weir GC (1984) Abnormal glucose regulation of insulin secretion in models of reduced $\mathrm{B}$ cell mass. Diabetes 33:667-673

5. Matveyenko AV, Veldhuis JD, Butler PC (2006) Mechanisms of impaired fasting glucose and glucose intolerance induced by an approximate 50\% pancreatectomy. Diabetes 55:2347-2356

6. Meier JJ, Kjems LL, Veldhuis JD, Lefèbvre P, Butler PC (2006) Post prandial suppression of glucagon secretion depends on intact pulsatile insulin secretion: further evidence for the intraislet insulin hypothesis. Diabetes 55:1051-1056

7. Ritzel RA, Butler AE, Rizza RA, Veldhuis JD, Butler PC (2006) Relationship between beta-cell mass and fasting blood glucose concentration in humans. Diabetes Care 29:717-718

8. Kjems LL, Kirby BM, Welsh EM et al (2001) Decrease in betacell mass leads to impaired pulsatile insulin secretion, reduced postprandial hepatic insulin clearance, and relative hyperglucagonemia in the minipig. Diabetes 50:2001-2012

9. Larsen MO, Wilken M, Gotfredsen CF, Carr RD, Svendsen O, Rolin B (2002) Mild streptozotocin diabetes in the Gottingen minipig. A novel model of moderate insulin deficiency and diabetes. Am J Physiol Endocrinol Metab 282:E1342-E1351

10. Kendall DM, Sutherland DE, Najarian JS, Goetz FC, Robertson RP (1990) Effects of hemipancreatectomy on insulin secretion and glucose tolerance in healthy humans. N Engl J Med 322:898-903

11. Bonner-Weir S, Baxter LA, Schuppin GT, Smith FE (1993) A second pathway for regeneration of adult exocrine and endocrine pancreas. A possible recapitulation of embryonic development. Diabetes 42:1715-1720

12. Peshavaria M, Larmie BL, Lausier J et al (2006) Regulation of pancreatic beta-cell regeneration in the normoglycemic $60 \%$ partial-pancreatectomy mouse. Diabetes 55:3289-3298

13. Menge BA, Tannapfel A, Belyaev $O$ et al (2008) Partial pancreatectomy in adult humans does not provoke beta-cell regeneration. Diabetes 57:142-149

14. Jimenez RE, Fernandez-Del Castillo C, Rattner DW, Warshaw AL (2003) Pylorus-preserving pancreaticoduodenectomy in the treatment of chronic pancreatitis. World J Surg 27:1211-1216

15. Litwin J, Dobrowolski S, Orlowska-Kunikowska E, Sledzinski Z (2008) Changes in glucose metabolism after Kausch-Whipple pancreatectomy in pancreatic cancer and chronic pancreatitis patients. Pancreas 36:26-30

16. Slezak LA, Andersen DK (2001) Pancreatic resection: effects on glucose metabolism. World J Surg 25:452-460

17. Uhl W, Gloor B, Buchler MW (1999) Pancreatic surgery. Curr Opin Gastroenterol 15:410-419

18. Meier JJ, Nauck MA, Pott A et al (2006) Glucagon-like peptide 2 stimulates glucagon secretion, enhances lipid absorption, and inhibits gastric acid secretion in humans. Gastroenterology. 130:44-54

19. Meier JJ, Hücking K, Holst JJ, Deacon C, Schmiegel W, Nauck MA (2001) Reduced insulinotropic effect of gastric inhibitory polypeptide in first-degree relatives of patients with type 2 diabetes. Diabetes 50:2497-2504

20. Matsuda M, DeFronzo RA (1999) Insulin sensitivity indices obtained from oral glucose tolerance testing. Diabetes Care 22:1462-1470
21. Meier JJ, Gallwitz B, Askenas M et al (2005) Secretion of incretin hormones and the insulinotropic effect of Gastric Inhibitory Polypeptide (GIP) in women with a history of gestational diabetes. Diabetologia 48:1872-1881

22. Permert J, Adrian TE, Jacobsson P, Jorfelt L, Fruin AB, Larsson J (1993) Is profound peripheral insulin resistance in patients with pancreatic cancer caused by a tumor-associated factor. Am J Surg 165:61-66 discussion 66-7

23. Dugan MC, Sarkar FH (1998) Current concepts in pancreatic cancer: symposium summary. Pancreas 17:325-333

24. Permert J, Ihse I, Jorfeldt L, von Schenck H, Arnqvist HJ, Larsson J (1993) Pancreatic cancer is associated with impaired glucose metabolism. Eur J Surg 159:101-107

25. Horowitz M, Edelbroek MA, Wishart JM, Straathof JW (1993) Relationship between oral glucose tolerance and gastric emptying in normal healthy subjects. Diabetologia 36:857-862

26. Horowitz M, O'Donovan D, Jones KL, Feinle C, Rayner CK, Samsom M (2002) Gastric emptying in diabetes: clinical significance and treatment. Diabetic Med 19:177-194

27. Meier JJ, Gallwitz B, Salmen S et al (2003) Normalization of glucose concentrations and deceleration of gastric emptying after solid meals during intravenous glucagon-like peptide 1 in patients with type 2 diabetes. J Clin Endocrinol Metab 88:2719-2725

28. Young A (2005) Inhibition of gastric emptying. Adv Pharmacol 52:99-121

29. Fernandez-del Castillo C, Rattner DW, Warshaw AL (1995) Standards for pancreatic resection in the 1990s. Arch Surg 130:295-299 discussion 299-300

30. Jimenez RE, Fernandez-del Castillo C, Rattner DW, Chang Y, Warshaw AL (2000) Outcome of pancreaticoduodenectomy with pylorus preservation or with antrectomy in the treatment of chronic pancreatitis. Ann Surg 231:293-300

31. Kobayashi I, Miyachi M, Kanai M et al (1998) Different gastric emptying of solid and liquid meals after pylorus-preserving pancreatoduodenectomy. Br J Surg 85:927-930

32. Strommer L, Raty S, Hennig R et al (2005) Delayed gastric emptying and intestinal hormones following pancreatoduodenectomy. Pancreatology 5:537-544

33. Sato T, Imamura M, Matsuro S, Sasaki I, Ohneda A (1986) Gastric acid secretion and gut hormone release in patients undergoing pancreaticoduodenectomy. Surgery 99:728-734

34. Nyboe Andersen B, Krarup T, Thorsgaard Pedersen NT, Faber OK, Hagen C, Worning H (1982) B cell function in patients with chronic pancreatitis and its relation to exocrine pancreatic function. Diabetologia 23:86-89

35. Larsen S, Hilsted J, Tronier B, Worning H (1987) Metabolic control and B cell function in patients with insulin-dependent diabetes mellitus secondary to chronic pancreatitis. Metabolism 36:964-967

36. Permert J, Ihse I, Jorfeldt L, von Schenck H, Arnquist HJ, Larsson J (1993) Improved glucose metabolism after subtotal pancreatectomy for pancreatic cancer. Br J Surg 80:1047-1050

37. Donev S, Petkov P, Marquie G, Duhault J, Jablenska R (1989) Immunohistochemical investigations of the endocrine pancreas in normoglycemic sand rats (Psammomys obesus). Acta Diabetol Lat 26:309-313

38. Baetens D, Malaisse-Lagae F, Perrelet A, Orci L (1979) Endocrine pancreas: three-dimensional reconstruction shows two types of islets of Langerhans. Science 206:1323-1325

39. Yoon KH, Ko SH, Cho JH et al (2003) Selective beta-cell loss and alpha-cell expansion in patients with type 2 diabetes mellitus in Korea. J Clin Endocrinol Metab 88:2300-2308

40. Robertson RP, Lanz KJ, Sutherland DE, Seaquist ER (2002) Relationship between diabetes and obesity 9 to 18 years after hemipancreatectomy and transplantation in donors and recipients. Transplantation 73:736-741 
41. Kumar AF, Gruessner RW, Seaquist ER (2008) Risk of glucose intolerance and diabetes in hemipancreatectomized donors selected for normal preoperative glucose metabolism. Diabetes Care 9:9

42. Jalleh RP, Williamson RC (1992) Pancreatic exocrine and endocrine function after operations for chronic pancreatitis. Ann Surg 216:656-662
43. Miyata M, Takao T, Uozumi T, Okamoto E, Manabe H (1974) Insulin secretion after pancreatoduodenectomy. Ann Surg 179:494-498

44. Beger HG, Krautzberger W, Bittner R, Buchler M, Limmer J (1985) Duodenum-preserving resection of the head of the pancreas in patients with severe chronic pancreatitis. Surgery 97:467-473 